\title{
Vaccination contre le virus du papillome humain
}

\author{
Dans l'ensemble, le vaccin contre le virus du papillome humain (VPH) est bien toléré, \\ mais les mesures de précaution qui figurent dans l'information professionnelle doi- \\ vent être respectées.
}

Pia Caduff-Janosa

\section{La plupart des effets indésirables postvaccinaux notifiés ont connu une évolution bénigne et étaient autolimités}

Correspondance:

Dr méd. Pia Caduff-Janosa Swissmedic

Schweizerisches Heilmittelinstitut Abteilung Arzneimittelsicherheit Hallerstrasse 7 CH-3000 Berne 9 Tél. 0313238623

pia.caduff@swissmedic.ch
En Suisse, deux vaccins contre le virus du papillome humain (VPH) ont été autorisés et sont disponibles sur le marché: Cervarix ${ }^{\circledR}$ et Gardasil $^{\circledR}$. Ces deux produits sont utilisés pour prévenir l'apparition de tumeurs malignes des organes génitaux féminins dues aux types 16 et $18 \mathrm{du} \mathrm{VPH}$. Gardasi ${ }^{\circledR}$ est un vaccin quadrivalent (types 16,18 , 6 et $11 \mathrm{du} \mathrm{VPH}$ ) qui protège également contre les condylomes.

L'Office fédéral de la santé publique (OFSP) recommande ce vaccin, en général bien supporté, chez les filles à partir de l'âge de 11 ans. Au total, trois doses sont administrées par voie intramusculaire, séparées par un délai d'un mois entre la première et la deuxième injection et d'au moins trois mois entre la deuxième et la troisième. dant l'année scolaire 2009/2010. Cependant, nul ne connaît le nombre de jeunes femmes qui ont été vaccinées en dehors de ces programmes depuis 2006. Dans le monde, 61 millions de doses ont été distribuées.

La plupart des EIP notifiés en rapport avec Garda$\mathrm{sil}^{\circledR}$ ont connu une évolution bénigne et étaient autolimités. Ainsi, sur les 117 annonces reçues, seules 15 concernaient des EIP graves (requérant une hospitalisation par exemple), et 18 des EIP médicalement importants. Quatre de ces annonces ne mentionnaient pas l'évolution et trois patientes n'étaient pas encore rétablies au moment de l'envoi de l'annonce, mais les 26 autres femmes concernées avaient entièrement récupéré. Les EIP graves étaient les suivants: syncopes avec complications, fièvre avec symptômes concomitants dont la cause a été identifiée lors de l'hospitalisation, myalgies prolongées, arthralgies, et un cas d'encéphalomyélite (voir ci-dessous).

La moitié des réactions notifiées concernait deux classes d'organes (troubles du système nerveux et troubles généraux), et un tiers touchait le système digestif. Il s'agissait essentiellement de maux de tête, de vertiges, de nausées, de vomissements, de pâleur, de réactions fébriles et de syncopes. Une des syncopes a occasionné une chute ayant entraîné une fracture du crâne et une hémorragie sous-arachnoïdienne minime. Il faut savoir que les syncopes lors de vaccinations chez des adolescents sont des événements bien documentés [1], raison pour laquelle la vaccination doit être administrée en position couchée ou assise et suivie d'une période d'observation d'au moins 15 minutes.

Certaines annonces font état de réactions allergiques bénignes et auto-limitées. Swissmedic n'a reçu aucune notification de réactions anaphylactiques, mais la banque de données de l'OMS, qui contient plus de cinq millions d'annonces sur des effets indésirables de médicaments (vaccins y compris) survenus dans le monde entier, comporte plusieurs rapports mentionnant ce type de réaction. Selon certaines données australiennes [2], il s'agit là d'un phénomène très rare, mais sa fréquence de 2,6 pour 100000 doses 
(95\% IC, 1,0-5,3 pour 100000) est un peu plus élevée que celle des autres vaccins. Une incidence de 0 à 3,5 par million de doses a été également relevée lors d'études antérieures concernant d'autres vaccins ayant été prescrits à des enfants et des adolescents. Cependant, sachant que tous les médicaments administrés par voie parentérale peuvent en principe déclencher une réaction allergique grave, l'équipement adéquat devrait être prêt et les personnes vaccinées devraient être surveillées de manière à pouvoir prendre immédiatement les mesures thérapeutiques nécessaires le cas échéant.

\section{L'institut quant à lui n'a reçu aucune annonce de décès}

Un rapport faisait état de l'apparition d'une encéphalite du tronc cérébral avec paralysie du nerf moteur oculaire externe environ un mois après la deuxième dose de Gardasil ${ }^{\circledR}$. Mais les données disponibles ne permettent pas de déterminer s'il s'agit en l'occurrence des premières manifestations d'une sclérose en plaques ou si ces symptômes ont été déclenchés par la vaccination. Dans la banque de données de l'OMS, 26 effets indésirables sur les 13108 recensés au sujet de tous les vaccins contre le VPH sont des scléroses en plaques. A l'échelle internationale, certains cas isolés d'encéphalomyélites aiguës disséminées ont été notifiés après l'administration de Gardasil ${ }^{\circledR}$.

Un autre rapport porte sur une thrombose veineuse au niveau du mollet chez une jeune femme dont l'anamnèse ne comportait aucun facteur de risque. L'on n'a pas encore déterminé si la jeune femme souffrait éventuellement de troubles de la coagulation. La FDA, autorité de contrôle des médicaments aux EtatsUnis, a également reçu des annonces d'événements thrombo-emboliques veineux après injection de vaccins contre le VPH. Mais 90\% des femmes concernées présentaient des facteurs de risque pré-existants de thrombo-embolies veineuses.
En 2008, la presse a mentionné des cas de décès à la suite de l'administration de vaccins contre le VPH. La banque de données de l'OMS en recense quatre, et la FDA en a enregistré 32 jusqu'à fin 2008: huit rapports n'ont pu être confirmés, vingt décès ont été attribués à des maladies graves pré-existantes, et les quatre décès restants n'ont pu être élucidés [3]. L'institut quant à lui n'a reçu aucune annonce de décès.

Début février 2009, deux cas de crises convulsives graves et prolongées ont été notifiés en Espagne suite à la vaccination contre le VPH. Sachant que les doses administrées aux deux jeunes filles provenaient du même lot, la qualité des vaccins a été vérifiée de manière approfondie sans qu'aucune déviation ne soit constatée. L'EMA, l'autorité européenne de contrôle de médicaments, a jugé le lien de causalité entre ces crises convulsives et le vaccin improbable.

Swissmedic surveille constamment le profil de sécurité de tous les vaccins; nous vous invitons donc à annoncer les effets indésirables que vous avez observés (y compris lorsqu'il s'agit de présomptions) aux centres régionaux de pharmacovigilance en utilisant le formulaire jaune correspondant. Ce dernier peut également être rempli sur ordinateur et envoyé par voie électronique. Il est disponible sur le site Internet de Swissmedic (www.swissmedic.ch $\rightarrow$ Annonce d'effets indésirables), dans le Compendium suisse des médicaments, et auprès de Swissmedic ou des centres régionaux de pharmacovigilance.

\section{Références}

1 Braun MM, Patriarca PA, Ellenberg SS. Syncope after immunization. Arch Pediatr Adolesc Med. 1997;151:255-9.

2 Brotherton JML, Gold MS, Kemp AS et al. Anaphylaxis following quadrivalent human papillomavirus vaccination. CMAJ. 2008;179:509-10.

3 Slade BA, Leidel L, Vellozzi C et al. Postlicensure safety surveillance for quadrivalent human papillomavirus recombinant vaccine. JAMA. 2009;302(7):750-7. 